\title{
Question Answering Pilot Task at CLEF 2004
}

\author{
Jesús Herrera, Anselmo Peñas, and Felisa Verdejo \\ Departamento de Lenguajes y Sistemas Informáticos, \\ Universidad Nacional de Educación a Distancia, \\ Juan del Rosal, 16, E-28040 Madrid, Spain \\ \{jesus.herrera, anselmo, felisa\}@lsi.uned.es
}

\begin{abstract}
A Pilot Question Answering Task has been activated in the Cross-Language Evaluation Forum 2004 with a twofold objective. In the first place, the evaluation of Question Answering systems when they have to answer conjunctive lists, disjunctive lists and questions with temporal restrictions. In the second place, the evaluation of systems' capability to give an accurate self-scoring about the confidence on their answers. In this way, two measures have been designed to be applied on all these different types of questions and to reward systems that give a confidence score with a high correlation with the human assessments. The forty eight runs submitted to the Question Answering Main Track have been taken as a case of study, confirming that some systems are able to give a very accurate score and showing how the measures proposed reward this fact.
\end{abstract}

\section{Introduction}

A Pilot Question Answering (QA) Task has been activated this year within the Main QA Track of the CLEF ${ }^{1} 2004$ campaign. The Pilot Task aims at investigating how QA systems are able to cope with types of questions different from the ones posed in the Main Track. To accomplish it, a set of questions has been prepared and new evaluation measures have been proposed.

Few questions were similar to those posed in the Main Track (factoid and definition questions) although they were selected with more than one correct and distinct answer. Questions whose answer is a list of items were also posed, following TREC ${ }^{2}$ and $\mathrm{NTCIR}^{3}$ previous experiences. Finally, more than half of the questions in the Pilot Task aim at dealing with temporal restrictions.

The evaluation measure proposed for this Pilot Task has been designed to take into consideration all these types of questions and, simultaneously, reward systems that, even focusing their attention on a few types of questions, are

\footnotetext{
${ }^{1}$ Cross-Language Evaluation Forum, http://www.clef-campaign.org

2 Text REtrieval Conference, http://trec.nist.gov

${ }^{3}$ NII-NACSIS Test Collection for IR Systems, http://research.nii.ac.jp/ntcir/indexen.html 
able to obtain very accurate results, with a good answer validation and a good confidence score.

In the present edition, the Pilot Task has been activated only for Spanish and has been carried out simultaneously with the Main QA Track. Participants in the Pilot Task have made a special effort to accomplish the extra work.

Section 2 describes the task and the different types of questions, including those with temporal restrictions. Section 3 presents some criteria to design the evaluation measure and presents the $K$ and $K 1$ measures. The results for the Main QA Track at CLEF [3] are taken as a case of study to discuss and compare these measures with the previous ones used at TREC, NTCIR and CLEF. Section 4 presents the results obtained by the participants in the Pilot Task and, finally, Section 5 points out some conclusions and future work.

\section{Task Definition}

The QA Pilot Task followed the rules stated in the QA Main Track guidelines except for the source and the target languages, the type and number of questions, and the evaluation measure.

One hundred of questions were posed in Spanish and the corpus used was the EFE Spanish press agency collection of news from 1994 and 1995. The questions of this Pilot Task were distributed among the following types: factoid (18), definition (2), conjunctive list (20), temporally restricted by date (20), temporally restricted by period (20), and temporally restricted by event (20 nested questions). A little amount of questions had no answer in the document collection (2 NIL factoid questions). As usual, a question was assumed to have no answer when neither human assessors nor participating systems could find one.

Ideally, QA systems should tend to give only one answer for each question but, however, there exist some questions whose answer depends on the context or changes in time. In these cases, disjunctive lists are obtained, that is, lists of different and correct items representing a disjunction of concepts. The decision of which one of them is the most correct is strongly dependent on the user's information need, text errors, consistency between different texts (specially in the news domain), etcetera. Therefore, being able to obtain all the possible correct and distinct answers to a question seems to be a desirable feature for open domain QA systems.

For this reason, there was no limit to the number of answers at the Pilot Task, but at least one answer to each question had to be given. If systems believed that it was no response to a question in the corpus, they had to answer NIL.

In the conjunctive list type of questions, a determined or undetermined quantity of items is required to set up a single answer. For the Pilot Task, the goal was to obtain the largest amount of different items within each answer.

Three subtypes of temporally restricted questions have been proposed at the Pilot Task (by date, by period and by event), and three moments with regard to the restriction (before, during or after the temporal restriction): 
- Restriction by Date, where a precise date contextualises the question, which can refer either to a particular moment, or to a time before or after it. A date could consist in a day, a month, a year, etcetera, depending on the question. Examples:

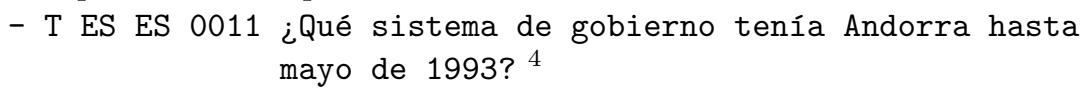

- T ES ES 0014 ¿Quién visitó Toledo el 22 de febrero de 1994? ${ }^{5}$

- Restriction by Period. In this case, questions are referred explicitly to a whole period or range of time. A period could be expressed by a pair of dates delimiting it, or by a name accepted as designation of some important periods as, for example, Cuaresma ${ }^{6}$. Examples:

- T ES ES 0086 ¿Quién reinó en España durante el Siglo de Oro de su literatura? ${ }^{7}$

- T ES ES 0037 ¿Quién gobernó en Bolivia entre el 17 de julio de 1980 y el 4 de agosto de 1981 ? $^{8}$

- Event restriction, that implies an embedded or implicit extra question so that it is necessary to answer the nested question to determine the temporal restriction. Then, the temporal restriction refers to the moment in which the second event occurred. For example:

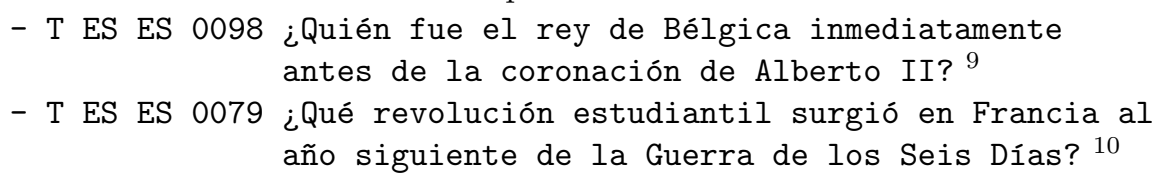

The degree of inference necessary to solve the temporal restrictions was not the same for all the questions. In some questions a reference to the temporal restriction could be found in the same document, while in other questions it was necessary to access other documents to temporally locate the question.

\section{Evaluation Measure}

The evaluation measure has been designed in order to reward systems that return as many different and correct answers as possible to each question but, at the same time, punishing at the same time the incorrect answers. Two reason motivate the penalization of incorrect answers: First, it is assumed that a user of a QA system would prefer a void answer rather than an incorrect one. Systems must validate their answers and must give an accurate confidence score. Second,

\footnotetext{
${ }^{4}$ What kind of government did Andorra have until May 1993?

${ }^{5}$ Who visited Toledo on 22nd February 1994 ?

${ }^{6}$ Cuaresma is the Spanish word for Lent.

${ }^{7}$ Who reigned in Spain during what is called in the literature "The Golden Age"?

${ }^{8}$ Who governed Bolivia between 17th July 1980 and 4th August 1981?

${ }^{9}$ Who was the king of Belgium just before the coronation of Albert II?

${ }^{10}$ What student revolution took place in France in the year after the Six-Day War?
} 
since there was no limit in the number of answers, systems had to prevent the risk of giving too much incorrect ones. The effect was that no more than three answers per question were given.

In order to evaluate systems' self-scoring, a mandatory confidence score represented by a real number ranged between 0 and 1, was requested. 0 meant that the system had no evidence on the correctness of the answer, and 1 meant that the system was totally sure about its correctness.

The evaluation measure has been designed to reward systems that:

- answer as many questions as possible,

- give as many different right answers to each question as possible,

- give the smallest number of wrong answers to each question,

- assign higher values to right answers,

- assign lower values to wrong answers,

- give answer to questions that have less known answers.

\subsection{The $K$-Measure}

According to the criteria above, the evaluation measure is defined as follows:

$$
\begin{gathered}
K(\text { sys })=\frac{1}{\# \text { questions }} \cdot \sum_{i \in q u e s t i o n s} \frac{\sum_{r \in \text { answer }(\text { sys }, i)} \operatorname{score}(r) \cdot \operatorname{eval}(r)}{\max \{R(i), \text { answered }(\text { sys }, i)\}} \\
K(\text { sys }) \in \mathbb{R} \wedge K(\text { sys }) \in[-1,1]
\end{gathered}
$$

where $R(i)$ is the total number of known answers to the question $i$ that are correct and distinct; answered(sys,i) is the number of answers given by the system sys for the question $i$; score $(r)$ is the confidence score assigned by the system to the answer $r$; eval ( $r$ ) depends on the judgement given by a human assessor.

$$
\text { eval }(r)=\left\{\begin{array}{clll}
1 & \text { if } & r & \text { is judged as correct } \\
0 & \text { if } & r & \text { is a repeated answer } \\
-1 & \text { if } & r & \text { is judged as incorrect }
\end{array}\right.
$$

When $K($ sys $)$ equals 0 it matches with a system without knowledge that assigns 0 to the confidence score of all their answers. Therefore, $K($ sys $)=0$ is established as a baseline and $K$-measure gives an idea about the system's knowledge.

The answer finding process, accomplished by human assessors, is strongly determined by the evaluation measure. In the case of $K$-measure the parameter $R(i)$ requires a knowledge of all the correct and distinct answers contained in the corpus for each question. This fact introduces a very high cost in the preassessment process because it is not easy to ensure that, even with a human search, all distinct answers for each question have been found in a very large corpus. One alternative is to make the pre-assessment process less strict and consider only the set of different answers found by humans or systems along the process. Another alternative is to request only one answer per question and ignore recall. 


\subsection{The $K 1$-Measure}

A second measure, derived from the $K$-measure, is proposed to evaluate exercises when just one answer per question is requested (number of questions equals number of answers) or when the achievement of all the possible answers by the system is not outstanding for the exercise. That measure has been called $K 1$ measure ( $K$-measure for systems giving 1 answer per question) and it is defined as follows:

$$
\begin{gathered}
K 1(\text { sys })=\frac{\sum_{r \in \text { answers }(\text { sys })} \text { score }(r) \cdot \operatorname{eval}(r)}{\# \text { questions }} \\
K 1(\text { sys }) \in \mathbb{R} \wedge K 1(\text { sys }) \in[-1,1]
\end{gathered}
$$

where score $(r)$ is the confidence score assigned by the system to the answer $r$ and eval $(r)$ depends on the judgement given by a human assessor.

$$
\operatorname{eval}(r)=\left\{\begin{array}{cl}
1 & \text { if } r \text { is judged as correct } \\
-1 & \text { in other case }
\end{array}\right.
$$

Again, $K 1($ sys $)=0$ is established as a baseline.

\subsection{Comparison with Precedent Measures}

Comparing $K$ and $K 1$ measures with other measures used in precedent QA evaluation exercises, the following differences and similarities are found:

- Accuracy measure, commonly used in all QA evaluations [1] [2] [4] [7] [8] [9] [10] [11], measures the precision in giving correct answers. But it does not take into account the confidence score, as in $K$ and $K 1$ measures, nor the recall when more than one answer per question is given, as in F-measure or $K$-measure.

- Mean F-measure, used in the QA Track at TREC 2003 [11] and in the QA Challenge at NTCIR 2002 [1], gives a combination between precision and recall, generally the mean of both. As the $K$-measure, it is designed for systems that must give all the correct answers existing in the corpus for every question. The $K$-measure takes into account a combination of precision and recall by means of the $\max \{R(i)$, answered $(s y s, i)\}$ denominator. In addition, $K$ and $K 1$ measures include the confidence score into their calculations.

- Mean Reciprocal Rank, used in the QA Track at TREC [7] [8] [9] [10], in the QA Challenge at NTCIR 2002 [1] and in the QA Track at CLEF 2003 [2] [4]. It is designed for systems that give one or more answers per question, in a decreasing order of confidence. It rewards systems assigning a higher confidence to the correct answers. However, Mean Reciprocal Rank cannot evaluate systems that find several different and correct answers for the same question, and the incorrect answers are not considered as a worse case than the absence of answers.

- Confident-Weighted Score (CWS), used in the QA Track at TREC 2002 [10] and in the QA Track at CLEF 2004 [3] as a secondary measure. It is 
designed for systems that give only one answer per question. Answers are in a decreasing order of confidence and CWS rewards systems that give correct answers at the top of the ranking. Hence, correct answers in the lower zone of the ranking make a very poor contribution to the global valuation, and this contribution is determined by the ranking position instead of the system's self-scoring.

\subsection{Correlation Between Self-scoring and Correctness}

Since the confidence score has been included in the $K$-measure, a high correlation between self-scoring and correctness is expected to produce higher values of $K$. However, it is interesting to know separately the quality of the scoring given by every system. Hence, it is proposed the use of the correlation coefficient $(r)$ between self-scoring value (in range $[0,1]$ ) and the value associated to the human assessment: 1 for the correct answers and 0 otherwise. That is:

$$
\begin{gathered}
r(\text { sys })=\frac{\sigma_{\text {assess }(\text { sys }) \text { score }(\text { sys })}}{\sigma_{\text {assess }(\text { sys })} \cdot \sigma_{\text {score }(\text { sys })}} \\
r(\text { sys }) \in \mathbb{R} \wedge r(\text { sys }) \in[-1,1]
\end{gathered}
$$

where assess(sys) and score(sys) are the two multidimensional variables containing the values of the human assessment and the confidence score for the system sys, respectively; $\sigma_{\text {assess(sys) }}, \sigma_{\text {score(sys) }}$ are the typical deviations for assess(sys) and score(sys), respectively, and $\sigma_{\text {assess(sys)score(sys) }}$ is the covariance between the two variables.

When a system assigns a score $=1$ to its correct answers and score $=0$ to the rest, it obtains a correlation coefficient $r=1$, meaning that such a system has a perfect knowledge about the correctness of its response. A correlation coefficient equal to 0 indicates that score and correctness have no correlation. A negative value indicates that there is a certain correlation but in the opposite direction.

\subsection{A Case of Study}

In the QA 2004 Main Track [3], the confidence score has been requested in order to calculate the CWS as a secondary evaluation measure. This confidence score, together with the human assessments of all the submitted runs, permitted to study the effect of the $K 1$-measure in the ranking of systems, and to compare the official measures with this one. No conclusions should be stated about the quality of systems because they should not be compared across different target languages, and also because they did not develop any strategy in order to obtain good values of $K 1$. However, evaluation measures are evaluated here, not systems.

Table 1 shows the number of given correct answers, CWS, $K 1$ and the correlation coefficient for all the systems participating in the QA at CLEF 2004 Main Track.

A higher correlation coefficient (higher score for the correct answers) brings associated better values of $K 1$ for the same or similar number of given correct answers. For example, ptue041ptpt, with the higher correlation coefficient $(r>$ 
Table 1. Values and rankings for accuracy, CWS, K1, and correlation coefficient r, for all runs submitted to the Main QA Track at CLEF 2004

\begin{tabular}{|c|c|c|c|c|c|c|c|c|}
\hline \multirow[b]{2}{*}{ run } & \multicolumn{3}{|c|}{ correct answers } & \multicolumn{2}{|c|}{ CWS } & \multicolumn{2}{|c|}{$\bar{K} 1$} & \multirow[b]{2}{*}{$\mathbf{r}$} \\
\hline & \# & $\%$ & ranking & value & ranking & value & ranking & \\
\hline uams042nlnl & 91 & 45.50 & 1 & 0.3262 & 2 & 0.0078 & \begin{tabular}{c|}
2 \\
\end{tabular} & 0.1148 \\
\hline uams041nlnl & 88 & 44 & 2 & 0.2841 & 3 & 0.0063 & 3 & 0.0987 \\
\hline uams041ennl & 70 & 35 & 3 & 0.2222 & 4 & 0.0055 & 4 & 0.1105 \\
\hline fuha041dede & 67 & 33.50 & 4 & 0.3284 & 1 & -0.3271 & 28 & 0.0094 \\
\hline aliv042eses & 65 & 32.50 & 5 & 0.1449 & 8 & -0.0416 & 15 & 0.1711 \\
\hline aliv041eses & 63 & 31.50 & 6 & 0.1218 & 9 & -0.0500 & 16 & 0.1099 \\
\hline irst041itit & 56 & 28 & 7 & 0.1556 & 7 & -0.1853 & 19 & 0.2128 \\
\hline talp042eses & 52 & 26 & 8 & 0.1029 & 13 & -0.2252 & 20 & -0.0366 \\
\hline dfki041dede & 51 & 25.50 & $9 . .10$ & $\mathrm{~N} / \mathrm{A} \dagger$ & $\mathrm{N} / \mathrm{A}$ & 0 & $5 . .14$ & $\mathrm{~N} / \mathrm{A}$ \\
\hline ilcp041itit & 51 & 25.50 & $9 . .10$ & $\mathrm{~N} / \mathrm{A}$ & $\mathrm{N} / \mathrm{A}$ & 0 & $5 . .14$ & $\mathrm{~N} / \mathrm{A}$ \\
\hline gine042frfr & 49 & 24.50 & 11 & 0.1140 & 11 & -0.2748 & 23 & -0.0339 \\
\hline talp041eses & 48 & 24 & 12 & 0.0878 & 15 & -0.2464 & 22 & -0.0483 \\
\hline ptue041ptpt & 47 & 23.62 & 13 & 0.2162 & 5 & 0.0201 & 1 & 0.5169 \\
\hline dfki041deen & 47 & 23.50 & 14 & 0.1771 & 6 & -0.5131 & 45 & -0.0453 \\
\hline inao041eses & 45 & 22.50 & $15 . .16$ & $\mathrm{~N} / \mathrm{A}$ & $\mathrm{N} / \mathrm{A}$ & 0 & $5 . .14$ & $\mathrm{~N} / \mathrm{A}$ \\
\hline irst041iten & 45 & 22.50 & $15 . .16$ & 0.1215 & 10 & -0.2310 & 21 & 0.1411 \\
\hline irst042itit & 44 & 22 & 17 & 0.1075 & 12 & -0.3248 & 27 & -0.0188 \\
\hline edin042fren & 40 & 20 & 18 & 0.0589 & 21 & -0.4066 & 38 & 0.0004 \\
\hline lire042fren & 39 & 19.50 & 19 & 0.0754 & 17 & -0.1738 & 18 & 0.3707 \\
\hline dltg041fren & 38 & 19 & 20 & $\mathrm{~N} / \mathrm{A}$ & $\mathrm{N} / \mathrm{A}$ & 0 & $5 . .14$ & $\mathrm{~N} / \mathrm{A}$ \\
\hline inao042eses & 37 & 18.50 & 21 & $\mathrm{~N} / \mathrm{A}$ & $\mathrm{N} / \mathrm{A}$ & 0 & $5 . .14$ & $\mathrm{~N} / \mathrm{A}$ \\
\hline irst042iten & 35 & 17.50 & 22 & 0.0751 & 18 & -0.3300 & 29 & 0.0566 \\
\hline edin042deen & 34 & 17 & $23 . .25$ & 0.0527 & 27 & -0.3556 & 31 & 0.1124 \\
\hline gine042defr & 34 & 17 & $23 . .25$ & 0.0970 & 14 & -0.2812 & 24 & -0.0371 \\
\hline gine042esfr & 34 & 17 & $23 . .25$ & 0.0750 & 19 & -0.3442 & 30 & -0.0282 \\
\hline edin041fren & 33 & 16.50 & 26 & 0.0570 & 22 & -0.5336 & 46 & -0.0560 \\
\hline dltg042fren & 29 & 14.50 & $27 . .31$ & $\mathrm{~N} / \mathrm{A}$ & $\mathrm{N} / \mathrm{A}$ & 0 & $5 . .14$ & $\mathrm{~N} / \mathrm{A}$ \\
\hline gine041defr & 29 & 14.50 & $27 . .31$ & 0.0790 & 16 & -0.3747 & 34 & -0.0471 \\
\hline gine042itfr & 29 & 14.50 & $27 . .31$ & 0.0540 & 26 & -0.3948 & 37 & -0.0467 \\
\hline gine042nlfr & 29 & 14.50 & $27 . .31$ & 0.0650 & 20 & -0.3682 & 33 & -0.0507 \\
\hline gine042ptfr & 29 & 14.50 & $27 . .31$ & 0.0560 & $24 . .25$ & -0.3818 & 35 & -0.0359 \\
\hline edin041deen & 28 & 14 & 32 & 0.0492 & 30 & -0.5515 & 47 & -0.0077 \\
\hline gine041frfr & 27 & 13.50 & $33 . .35$ & 0.0480 & 32 & -0.4425 & 41 & 0.0099 \\
\hline gine041esfr & 27 & 13.50 & $33 . .35$ & 0.0560 & $24 . .25$ & -0.4463 & 43 & 0.0991 \\
\hline gine042enfr & 27 & 13.50 & $33 . .35$ & 0.0510 & 29 & -0.3184 & 25 & -0.0336 \\
\hline bgas041bgen & 26 & 13 & 36 & 0.0564 & 23 & -0.3618 & 32 & 0.2023 \\
\hline gine041itfr & 25 & 12.50 & $37 . .38$ & 0.0490 & 31 & -0.3926 & 36 & -0.0368 \\
\hline gine041ptfr & 25 & 12.50 & $37 . .38$ & 0.0440 & $34 . .35$ & -0.4412 & 40 & -0.0595 \\
\hline sfnx042ptpt & 22 & 11.06 & $39 . .41$ & $\mathrm{~N} / \mathrm{A}$ & $\mathrm{N} / \mathrm{A}$ & 0 & $5 . .14$ & $\mathrm{~N} / \mathrm{A}$ \\
\hline cole041eses & 22 & 11 & $39 . .41$ & $\mathrm{~N} / \mathrm{A}$ & $\mathrm{N} / \mathrm{A}$ & 0 & $5 . .14$ & $\mathrm{~N} / \mathrm{A}$ \\
\hline lire041fren & 22 & 11 & $39 . .41$ & 0.0330 & 38 & -0.3200 & 26 & 0.2625 \\
\hline hels041fien & 21 & 10.61 & 42 & 0.0443 & 33 & -0.1136 & 17 & 0.0359 \\
\hline gine041nlfr & 20 & 10 & 43 & 0.0440 & $34 . .35$ & -0.4438 & 42 & -0.0369 \\
\hline mira041eses & 18 & 9 & $44 . .45$ & $\mathrm{~N} / \mathrm{A}$ & $\mathrm{N} / \mathrm{A}$ & 0 & $5 . .14$ & $\mathrm{~N} / \mathrm{A}$ \\
\hline gine041enfr & 18 & 9 & $44 . .45$ & 0.0333 & 37 & -0.4389 & 39 & -0.0349 \\
\hline sfnx041ptpt & 14 & 7.04 & 46 & $\mathrm{~N} / \mathrm{A}$ & $\mathrm{N} / \mathrm{A}$ & 0 & $5 . .14$ & $\mathrm{~N} / \mathrm{A}$ \\
\hline gine041bgfr & 13 & 6.50 & $47 . .48$ & 0.0514 & 28 & -0.5603 & 48 & -0.0181 \\
\hline gine $042 \mathrm{bgfr}$ & 13 & 6.50 & $47 . .48$ & 0.0380 & 36 & -0.4945 & 44 & 0.0928 \\
\hline
\end{tabular}

$0.5)$, has the 13 th position in the ranking for accuracy but reaches the 1st position for $K 1$.

On the contrary, there are some interesting examples, as fuha041dede or dfki041deen, that have a low or even negative correlation coefficient and experiment a huge drop in the ranking of $K 1$. 
However, these systems obtain a very good CWS value, showing that CWS does not reward a good correlation between self-scoring and correctness. Why do these systems obtain good values of CWS? The reason can be found looking at their answers in detail. When they have not enough confidence in the answer, they return NIL with a score 1, ensuring 20 correct answers (the 20 NIL questions) very high weighted in the CWS measure. All wrong NIL answers (up to 149, with score 1) affect negatively the correlation coefficient and also the $K 1$-measure. Somehow, they tuned their score to obtain a better CWS and, obviously, not a better $K 1$. Adopting a $K 1$ oriented strategy, they would obtain very good results. For example, if all NIL answers of fuha041dede had a score equal to 0 then the correlation coefficient would have been very high $(r=0.7385)$ and the system would have reached again the first place in the ranking with $K 1=0.218$.

These systems are an example of how state-of-the-art systems can give a very accurate self-scoring.

Since $K 1$ depends on the number of correct given answers, a good correlation coefficient is not enough to obtain good results: the more correct answers are given, the more positive components conform the global calculation of K1. For example, to beat fuha041dede using the mentioned $K 1$-oriented strategy $(K 1=$ $0.218)$, a system with perfect scoring $(\mathrm{r}=1)$ would need to answer correctly more than 40 questions (20\%).

\section{Results of the Pilot Task}

The data from the assessment process for the Pilot Task are shown in Table 2. Only one run from the University of Alicante (UA) [6] was submitted and, therefore, a comparison with other participants cannot be done. The UA system is based on the splitting of nested questions in order to answer questions with temporal restrictions. The UA team has evaluated its system over the TERQAS corpus [5], obtaining better results than in this Pilot Task at CLEF 2004.

The UA system has correctly answered $15 \%$ of the questions. The best result corresponds to factoid questions with $22.22 \%$ of questions with a correct answer. However, in the past edition of QA at CLEF, this team obtained better results

Table 2. Results of the assessment process for the Pilot Task at CLEF 2004. Data from the run of the University of Alicante

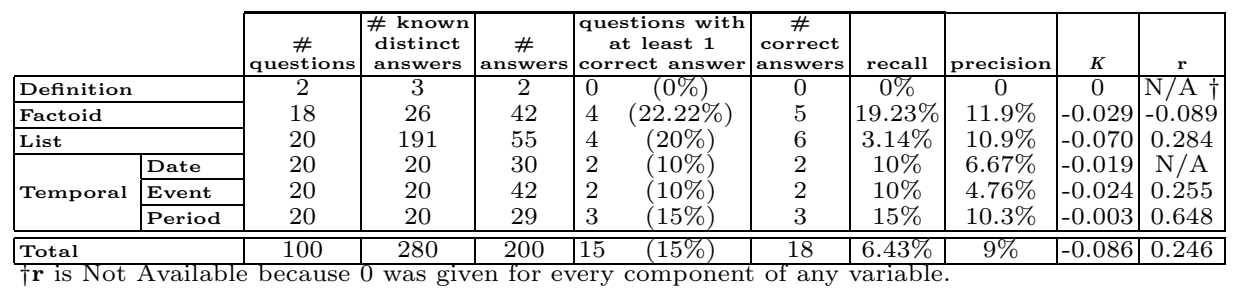


(up to $40 \%$ of questions with a correct answer) [2]. This results show that the questions posed in the Pilot Task were too difficult.

The UA system never gave more than three answers per question, independently of the type of formulated question. It seems an heuristically established limit for the system that has affected the achievement of good conjunctive and disjunctive list answers.

41 questions got NIL as an answer, with a confidence score of 0 for all them. Unfortunately, these 41 questions had at least one answer in the corpus. On the other hand, the UA system did not identify the 2 posed NIL questions.

Finally, it seems that the UA system did not manage the score value in the best way. The maximum value given for the confidence score was 0.5002 and several questions with only one correct answer in the corpus had associated several different answers with similar confidence score. The $K$-measure for the UA's exercise was $K=-0.086$ with a correlation coefficient of $r=0.246$ between self-scoring and real assessment.

\section{Conclusions}

Questions whose answer is a conjunctive or a disjunctive list, and questions with temporal restrictions, still remain a challenge for most QA systems. However, these are only a few types of difficult questions that QA systems will have to manage in the near future. A specialization and further collaboration among teams could be expected in order to achieve QA systems with higher accuracy and coverage for different types of questions. In fact, the QA Main Track at CLEF [3] shows that different participant systems answer correctly different subsets of questions.

Two measures have been proposed in order to reward systems that give a confidence score with a high correlation with human assessments and, at the same time, return more correct answers and less incorrect ones. The case of study shows that systems are able to give very accurate self-scoring, and that the $K$ and $K 1$ measures reward it. However, systems do not need to respond all the questions to obtain good results, but to find a good balance between the number of correct answers and the accuracy of their confidence score.

On the one hand, this seems a good way to promote the development of more accurate systems with better answer validation. On the other hand, it is a good way of permitting some specialization, openingn the possibility of posing new types of questions, dealing with multilinguality and, at the same time, leaving the door open for new teams starting to develop their own systems.

\section{Acknowledgements}

We are grateful to Julio Gonzalo, from UNED-NLP Group, and Alessandro Vallin, from ITC-Irst (Italy), for their contributions to this work. In addition, we would like to thank the University of Alicante team for their effort in participating in the Pilot Task. 
This work has been partially supported by the Spanish Ministry of Science and Technology within the following projects: TIC-2002-10597-E Organization of a Competitive Task for QA Systems; TIC-2003-07158-C04 Answer Retrieval from Digital Documents, R2D2; and TIC-2003-07158-C04-02 Multilingual Answer Retrieval Systems and Evaluation, SyEMBRA.

\section{References}

1. Fukumoto, J., Kato, T., Masui, F.: Question Answering Challenge (QAC-1). An Evaluation of Question Answering Task at NTCIR Workshop 3. In Keizo Oyama, Emi Ishida, Noriko Kando, editors: Proceedings of the Third NTCIR Workshop on Research in Information Retrieval, Automatic Text Summarization and Question Answering. National Institute of Informatics (2003)

2. Magnini, B., Romagnoli, S., Vallin, A., Herrera, J., Peñas, A., Peinado, V., Verdejo, F. , de Rijke, M.: The Multiple Language Question Answering Track at CLEF 2003. In C. Peters, J. Gonzalo, M. Braschler, M. Kluck, editors: Comparative Evaluation of Multilingual Information Access Systems. Results of the CLEF 2003 Evaluation Campaign. Lecture Notes in Computer Science 3237 Springer (2004) 479-495

3. Magnini, B., Vallin, A., Ayache, C., Erbach, G., Peñas, A., de Rijke, M., Rocha, P., Simov, K., Sutcliffe, R.: Overview of the CLEF 2004 Multilingual Question Answering Track. In C. Peters, F. Borri, editors: Results of the CLEF 2004 Croos-Language System Evaluation Campaign. Working Notes for the CLEF 2004 Workshop. Bath, United Kingdom (2004)

4. Peñas, A., Herrera, J., Verdejo, F.: Spanish Question Answering Evaluation. In A. Gelbukh editor: Computational Linguistics and Intelligent Text Processing, CICLing 2004. Lecture Notes in Computer Science 2945 Springer (2004) 472-483

5. Pustejovsky, J., Belanger, L., Casta, J., Gaizauskas, R., Hanks, P., Ingria, B., Katz, G., Radev, D., Rumshisky, A., Sanfilippo, A., Sauri, R., Sundheim, B., Verhagen, M.: TERQAS Final Report. Technical Report, MITRE, http://www.cs.brandeis.edu/ jamesp/arda/time/readings.html (2002)

6. Saquete, E., Martínez-Barco, P., Muñoz, R., Vicedo, J.L.: Splitting Complex Temporal Questions for Question Answering Systems. Proceedings of the 42nd Meeting of the Association for Computational Linguistics (ACL'04), Main Volume (2004) $566-573$

7. Voorhees, E. M.: The TREC-8 Question Answering Track Report. In E. M. Voorhees, D. K. Harman, editors: Proceedings of the Eigthh Text REtrieval Conference (TREC 8). NIST Special Publication 500-246 (1999) 77-82

8. Voorhees, E. M.: Overview of the TREC-9 Question Answering Track. In E. M. Voorhees, D. K. Harman, editors: Proceedings of the Ninth Text REtrieval Conference (TREC 9). NIST Special Publication 500-249 (2000) 71-79

9. Voorhees, E. M.: Overview of the TREC 2001 Question Answering Track. In E. M. Voorhees, D. K. Harman, editors: Proceedings of the Tenth Text REtrieval Conference (TREC 2001). NIST Special Publication 500-250 (2001) 42-51

10. Voorhees, E. M.: Overview of the TREC 2002 Question Answering Track. In E. M. Voorhees, L. P. Buckland, editors: Proceedings of the Eleventh Text REtrieval Conference (TREC 2002). NIST Special Publication 500-251 (2002)

11. Voorhees, E. M.: Overview of the TREC 2003 Question Answering Track. In: Proceedings of the Twelfth Text REtrieval Conference (TREC 2003). NIST Special Publication 500-255 (2003) 54-68 